\title{
Der europäische Deliktsgerichtsstand und die gewerblichen Schutzrechte
}

\section{Art. 7 Nr. 2 Brüssel Ia-VO im Lichte der aktuellen Rechtsprechung}

\author{
Peter Kindler
}

Die internationale Durchsetzung von gewerblichen Schutzrechten in der EU hängt prozessual vor allem davon ab, ob für Klagen aus der Verletzung geistigen Eigentums ein Deliktsgerichtsstand nach Art. 7 Nr. 2 Brüssel Ia-VO eröffnet ist. Daran hat der Inhaber des Immaterialgüterrechts ein erhebliches Interesse, wird doch der deliktische Erfolgsort regelmäßig in seinem Sitzstaat liegen. Die Anerkennung und Vollstreckung des Urteils im Sitzstaat des Gegners ist meist gesichert (Art. 35 ff. Brüssel Ia-VO). Ein Prozess vor den Gerichten des Staats, in dem der mutmaßliche Verletzer ansässig ist (Art. 4 Brüssel Ia-VO), liegt daher nicht unbedingt im Interesse des Rechteinhabers - dies auch im Hinblick auf die Vermeidung des höheren Zeit- und Kostenaufwands, der mit Auslandsprozessen verbunden ist. Der nachfolgende Beitrag zeigt jüngste Tendenzen in der einschlägigen Rechtsprechung auf.

\section{Vorbemerkung}

Zuständigkeitsrechtlich fällt die außervertragliche Haftung - unter anderem für den hier interessierenden Bereich der Verstöße gegen gewerbliche Schutzrechte - unter Art. 7 Nr. 2 Brüssel Ia-VO. Danach kann eine Person, die ihren Wohnsitz im Hoheitsgebiet eines Mitgliedstaats hat, in einem anderen Mitgliedstaat verklagt werden:

Erstveröffentlichung in GRUR 2018, 1107-1115. Mit freundlicher Genehmigung des Verlags C.H. Beck. Der Beitrag beruht auf dem Vortrag auf dem 1. Bozner Wirtschaftsrechtstag über „Italienisches, europäisches und internationales Immaterialgüterrecht“ am 15.06.2018 im Merkantilgebäude, Bozen. Für wertvolle Hinweise dankt der Verf. Prof. Dr. Ansgar Ohly, LMU München.

P. Kindler $(\square)$

Lehrstuhl für Bürgerliches Recht, Handels- und Gesellschaftsrecht, Internationales Privatrecht und Rechtsvergleichung, Ludwig-Maximilians-Universität München, München, Deutschland E-Mail: peter.kindler@jura.uni-muenchen.de 
„2. wenn eine unerlaubte Handlung oder eine Handlung, die einer unerlaubten Handlung gleichgestellt ist, oder wenn Ansprüche aus einer solchen Handlung den Gegenstand des Verfahrens bilden, vor dem Gericht des Ortes, an dem das schädigende Ereignis eingetreten ist oder einzutreten droht."

Art. 7 Nr. 2 Brüssel Ia-VO enthält gleichzeitig eine Festlegung der örtlichen Zuständigkeit. Spezialregelungen, insbesondere aus dem Unionsmarkenrecht, ${ }^{1}$ können vorgehen.

Der Tatortsgerichtsstand beruht auf dem - aus verschiedenen mitgliedstaatlichen Regelungen ${ }^{2}$ übernommenen - Grundgedanken, dass der durch eine unerlaubte Handlung Verletzte seine Schadensersatzansprüche dort geltend machen darf, wo das Unrecht begangen wurde, sich ausgewirkt hat oder sich auszuwirken droht, ohne sein Recht am allgemeinen Gerichtsstand des Schädigers geltend machen zu müssen. ${ }^{3}$ Hinzukommt das Prinzip der Sach- und Beweisnähe: Das Gericht des Orts, an dem das schädigende Ereignis eingetreten ist, eignet sich wegen der Nähe zum Prozessstoff und der leichteren Beweisaufnahme in der Regel am besten, den Rechtsstreit zu entscheiden. ${ }^{4} \mathrm{Ob}$ das forum delicti commissi darüber hinaus ein besonderes kompetenzrechtliches Privileg des (vorgeblich) Geschädigten enthält, wird durch den $E u G H$ nicht einheitlich beantwortet; ${ }^{5}$ dagegen spricht vor allem, dass im Stadium der Zuständigkeitsprüfung die materielle Rechtsverletzung - und damit eine mögliche Wertungsgrundlage für die Privilegierung des Klägers - naturgemäß noch gar nicht feststeht. ${ }^{6}$

\footnotetext{
${ }^{1}$ VO (EG) Nr. 207/2009 des Rates über die Unionsmarke; zu neueren Urteilen über Gerichtsstandsfragen auf diesem Gebiet Kur, GRUR 2018, 358, zu BGH, GRUR 2018, 84 - Parfummarken (mit einer bedenklichen Einschränkung des Verletzungsgerichtsstands); Mansel/Thorn/Wagner, IPRax 2018, 121 (134 f.); Subjecki, EWS 2018, 72 (76 ff.); ferner zu Zuständigkeitsfragen auf dem Gebiet des unionalen Immaterialgüterrechts Honorati, Jahrbuch für Italienisches Recht 29 (2017), 3; Ohly, Jahrbuch für Italienisches Recht 29 (2017), 27; monografisch Hansen, Die internationale Zuständigkeit bei Verletzung von Unionsschutzrechten, 2018.

${ }^{2}$ Vgl. § $32 \mathrm{ZPO}$, Art. 20 c.p.c. (Italien).

${ }^{3}$ Stadler in Musielak/Voit, ZPO, 15. Aufl. 2018, Art. 7 Brüssel Ia-VO Rn. 16; D. Paulus in Paulus/ Peiffer/Peiffer, Europäische Gerichtsstands- und Vollstreckungsverordnung (Brüssel Ia): EuGVVO, 2017, Art. 7 Rn. 138.

${ }^{4}$ StRspr., EuGH, GRUR 2018, 108 Rn. 27 - Bolagsupplysningen ua/Svensk Handel, mit Bespr. von Hau, GRUR 2018, 163; NJW 2002, 3617 Rn. 27 - Verein für Konsumenteninformation/Karl Heinz Henkel, mit Bespr. von Michailidou, IPRax 2003, 223; Leible in Rauscher, EuIPR/EuZPR, 2. Aufl. 2016, Art. 7 Brüssel Ia-VO Rn. 103, verweist zusätzlich auf die fehlende Manipulierbarkeit nach Eintritt des schädigenden Ereignisses.

${ }^{5}$ Näher Stadler in Musielak/Voit (o.Fn. 3), Art. 7 Brüssel Ia-VO Rn. 16 mit Verw. auf EuGH, GRUR 2013, 98 Rn. 46 - Folien Fischer und Fofitec (eindeutig gegen den Gedanken des Opferschutzes im Kompetenzrecht); ebenso EuGH, GRUR 2018, 108 Rn. 39 - Bolagsupplysningen ua/ Svensk Handel; vgl. demgegenüber EuGH, GRUR 2012, 300 - eDate Advertising und Martinez, wo der Gerichtshof auf die besondere - kompetenzrechtliche - Schutzwürdigkeit des Opfers abstellt, das via Internet in seinem Persönlichkeitsrecht verletzt wird; zu diesen Friktionen in der Rspr. Hoffmann, ZZP 128 (2015), 465 (471).

${ }^{6}$ Ähnl. D. Paulus in Paulus/Peiffer/Peiffer (o.Fn. 3), Art. 7 Brüssel Ia-VO Rn. 139; Hau, GRUR 2018, 163.
} 


\section{Kollisionsrecht bei Verletzung von Rechten des geistigen Eigentums}

\subsection{Auslegungskonkordanz und sachliche Reichweite des Art. 8 Rom II-VO}

Für die Beurteilung der zuständigkeitsrechtlichen Fragen ist unter anderem die kollisionsrechtliche Rechtsprechung zur Bestimmung des Deliktsstatuts bei Verletzung von Rechten des geistigen Eigentums von Interesse. Die insoweit maßgebliche Rom II-VO ist nach ihrem Erwägungsrund 7 im Lichte der Brüssel Ia-VO auszulegen, ${ }^{7}$ und diese Auslegungskonkordanz gilt auch in umgekehrter Richtung. ${ }^{8}$

Art. 8 Rom II-VO regelt die Anknüpfung außervertraglicher Schuldverhältnisse, die aus der Verletzung von Rechten des geistigen Eigentums entstanden sind. Der Begriff „Rechte des geistigen Eigentums“ umfasst namentlich Urheberrechte ${ }^{9}$ und gewerbliche Schutzrechte (Erwägungsgrund 26). Der Anwendungsbereich des Art. 8 Rom II-VO beschränkt sich nicht auf unerlaubte Handlungen, sondern erstreckt sich auf alle außervertraglichen Schuldverhältnisse im Bereich des geistigen Eigentums (Art. 13 Rom II-VO). Die einheitliche Anknüpfung soll Qualifikationsprobleme vermeiden und den Anknüpfungsgleichklang mit konkurrierenden Ansprüchen (insbesondere aus ungerechtfertigter Bereicherung) gewährleisten.

Art. 8 I Rom II-VO verweist auf das Recht des Staats, in dem der Schutz beansprucht wird (lex loci protectionis), und verwirklicht damit das allgemein anerkannte Schutzlandprinzip. ${ }^{10}$ Dem entspricht auf der Ebene des Sachrechts das Territorialitätsprinzip, da Immaterialgüterrechte von einem Staat nur mit Wirkung für sein Gebiet bewilligt werden. ${ }^{11}$

Nicht näher einzugehen ist im Folgenden auf außervertragliche Schuldverhältnisse, die aus einer Verletzung von unionsweit einheitlichen Rechten des geistigen Eigentums entstanden sind. Insoweit ist das einschlägige Recht der Union anzuwenden, ergänzend das Recht des Staats, in dem die Verletzung begangen wurde (Art. 8 II Rom II-VO). Dies hat namentlich Bedeutung für Unionsmarken und Unionsgeschmacksmuster; eine besondere Kollisionsnorm für das internationale Patentrecht

\footnotetext{
${ }^{7}$ Der Verweis in Erwägungsgrund 7 auf die Brüssel I-VO ist seit Inkrafttreten der Brüssel Ia-VO auf Letztere zu beziehen, vgl. Art. 80 S. 2 Brüssel Ia-VO.

${ }^{8}$ D. Paulus in Paulus/Peiffer/Peiffer (o.Fn. 3), Vorb. Art. 7 Brüssel Ia-VO Rn. 8; Kindler, GS Unberath, 2015, 255 (261); Linke/Hau, Int. Zivilverfahrensrecht, 7. Aufl. 2018, Rn. 2.27; Kropholler/ von Hein, EuZPR, 9. Aufl. 2011, Einl. Rn. 74.

${ }^{9}$ BGH, GRUR 2015, 264 Rn. 23-25 - Hi Hotel II (Abschlussentscheidung zu EuGH, GRUR 2014, 599 - Hi Hotel).

${ }^{10}$ BGH, GRUR 2018, 935 Rn. 22 - goFit, mwN; Grünberger, ZVglRWiss 108 (2009), 134; Sack, WRP 2008, 845; Schack, FS Kropholler, 2008, 651

${ }^{11}$ Vgl. nur BGH, GRUR 2012, 621 Rn. 34 - OSCAR (,Nach dem im Immaterialgüterrecht maßgeblichen Territorialitätsprinzip richtet sich der Schutz der inländischen Kennzeichen der Kl. nach dem Recht des Schutzlandes und damit nach deutschem Recht“); krit. Peifer, IPRax 2013, 228 (230): ,zumindest missverständlich“.
} 
enthält Art. 5 III der Einheitspatent-VO (EU) Nr. 1257/2012 ${ }^{12}$. Parteiautonome Handlungsoptionen bestehen im Immaterialgüterkollisionsrecht nicht: Im gesamten Anwendungsbereich des Art. 8 Rom II-VO ist eine Rechtswahl nach Art. 14 Rom II-VO ausgeschlossen (Art. 8 III Rom II-VO). Damit wollte der EU-Gesetzgeber die öffentlichen Interessen im Schutzland an der Abgrenzung der Rechte des geistigen Eigentums vor Eingriffen durch eine andere - von den Parteien gewählten - Rechtsordnung bewahren. ${ }^{13}$

\subsection{Der wirtschaftlich relevante Bezug zum Schutzland als Anwendungsvoraussetzung des nationalen Markenrechts}

Das Schutzlandprinzip des Art. 8 I Rom II-VO sei anhand der $B G H$-Entscheidung im Fall „Oscar all'italiana“ verdeutlicht: ${ }^{14}$ Die Klägerin veranstaltet die jährlich in Hollywood/USA stattfindenden „Academy Awards“, umgangssprachlich „OscarVerleihung“ genannt, in deren Rahmen die „Oscar-Statuetten“ als Preise für herausragende Leistungen im Spielfilmbereich vergeben werden. Sie ist Inhaberin der eingetragenen deutschen Wortmarke „OSCAR“. Die Beklagte betreibt den italienischen Fernsehsender RAI mit den Programmen RAI uno, RAI due, RAI tre und RAI international. In den Programmen der Beklagten liefen Fernsehsendungen mit den Titeln „Oscar del vino“, „La Kore Oscar della Moda“, „Oscar TV“ und „Oscar della Musica“, die italienische Preisverleihungsveranstaltungen zum Inhalt hatten. Die italienischsprachigen Sendungen konnten in Deutschland über Satellit und über das Kabelnetz empfangen werden. Die Klägerin hat bei einem deutschen Gericht beantragt, die Beklagte nach § 14 II Nrn. 2 und 3, V MarkenG zu verurteilen, es zu unterlassen, im geschäftlichen Verkehr die Bezeichnung „Oscar“ im Zusammenhang mit der Übermittlung und Ausstrahlung audiovisueller Produktionen zu benutzen. ${ }^{15}$ Das deutsche Markenrecht findet laut $B G H$ hier grundsätzlich Anwendung, weil die Klägerin den Schutz ihrer Marke für Deutschland beansprucht (Art. 8 I Rom II-VO). Die entscheidende Urteilspassage zur Bestimmung des Schutzlands lautet wie folgt: ${ }^{16}$, ,[...] die hier in Rede stehenden Fernsehsendungen richten sich bestimmungsgemäß auch an die Verkehrskreise im Inland. [Die] Satellitenausstrahlung der italienischen Fernsehprogramme durch die Bekl. [ist] darauf gerichtet, das Verständnis und die Kenntnis der italienischen Kultur und Sprache in der Welt zu

\footnotetext{
${ }^{12}$ Dazu näher MüKoBGB/Drexl, Bd. 12, 7. Aufl. 2018, IntImmGR Rn. 130 ff.; ders., FS Coester-Waltjen, 2015, 361; über das Europäische Patent mit einheitlicher Wirkung und das Einheitliche Patentgericht insb. Ohly, Jahrbuch für Italienisches Recht 29 (2017), 27.

${ }^{13}$ Dazu mit Recht krit. MüKoBGB/Drexl (o. Fn. 12), IntImmGR Rn. 271.

${ }^{14}$ BGH, GRUR 2012, 621 Rn. 16 ff. - OSCAR; dazu Peifer, IPRax 2013, 228.

${ }^{15}$ Art. 5 MRRL [2008/95/EG]; in Italien Art. 20 Codice della proprietà industriale (c. p.i.) - Diritti conferiti dalla registrazione (del marchio d'impresa).

${ }^{16}$ BGH, GRUR 2012, 621 Rn. 21 - OSCAR.
} 
fördern, so dass sich die Programme [...] auch an die deutsche Bevölkerung richten. [Dass] die Sendungen allein in italienischer Sprache ohne deutsche Übersetzung gesendet worden seien, steht [...] der Annahme einer bestimmungsgemäßen Aussendung jedenfalls an all diejenigen Fernsehzuschauer in Deutschland nicht entgegen, die die italienische Sprache verstehen.“

Darüber hinaus verlangte das Gericht für die Anwendbarkeit des deutschen Markenrechts ,einen hinreichenden wirtschaftlich relevanten Inlandsbezug“ (commercial effect). ${ }^{17}$ Festzustellen ist ein derartiger Inlandsbezug laut $B G H$ durch eine Gesamtabwägung der Interessen der Parteien, in die neben dem Gewicht der Auswirkungen der Kennzeichenbenutzung auf die inländischen wirtschaftlichen Interessen des Zeicheninhabers auch einfließen muss, inwieweit es den Beklagten möglich und zumutbar war, Rechtsverletzungen im Inland zu vermeiden. ${ }^{18}$

Auf dieser Prüfungsstufe bestehen allerdings Zweifel, ob durch die Ausstrahlung der italienischen „Oscar“-Sendungen eine relevante inländische Verletzungshandlung vorliegt. ${ }^{19}$ Nach der „HOTEL MARITIME“-Rechtsprechung ${ }^{20}$ steht dem Interesse des Kennzeicheninhabers an einer Unterlassung der Markenverletzung das Interesse des angeblichen Verletzers gegenüber, Unterhaltungssendungen auch über Satelliten in mehrere Mitgliedstaaten ausstrahlen zu dürfen. Markenverwendungen in grenzüberschreitenden Satellitenausstrahlungen sind daher nicht generell als taugliche Verletzungshandlungen einzustufen; das widerspräche der EU-Dienstleistungsfreiheit.

\section{Der zuständigkeitsrechtliche Deliktsort bei der Verletzung von Immaterialgüterrechten (,,Ort des schädigenden Ereignisses ${ }^{6}$ )}

\subsection{Ubiquitätsprinzip}

Unter dem - zuständigkeitsbegründenden - Ort des schädigenden Ereignisses versteht der EuGH sowohl den Ort, an dem der Schaden eingetreten ist als auch den Ort des ursächlichen Geschehens. ${ }^{21}$ Dieses so genannte „Ubiquitätsprinzip“

\footnotetext{
${ }^{17}$ BGH, GRUR 2012, 621 Rn. 37 - OSCAR; dabei handelt es sich um eine sachrechtliche Begrenzung des internationalen Anwendungsbereichs allein des deutschen Markenrechts, schon wegen der Unvereinbarkeit einer derartigen - dann allseitigen - kollisionsrechtlichen Aussage mit Art. 8 Rom II-VO, aA Peifer, IPRax 2013, 228 (230 nach Fn. 28).

${ }^{18}$ BGH, GRUR 2012, 621 Rn. 37 - OSCAR.

${ }^{19}$ Krit. auch Berlit, GRUR-Prax 2012, 189.

${ }^{20} \mathrm{BGH}$, GRUR 2005, 431 - HOTEL MARITIME.

${ }^{21}$ StRspr, in neuerer Zeit etwa EuGH, EuZW 2004, 477 Rn. 16 - Kronhofer; D. Paulus in Paulus/ Peiffer/Peiffer (o.Fn. 3), Art. 7 Brüssel Ia-VO Rn. 183 f.; Stadler in Musielak/Voit (o. Fn. 3), Art. 7 Brüssel Ia-VO Rn. 19.
} 
umfasst sonach bei Distanzdelikten sowohl den Handlungs- wie auch den Erfolgsort. Ausweislich der neueren Rechtsprechung spielt diese Regel gerade bei grenzüberschreitenden Markenrechtsverletzungen eine gewisse Rolle: Auch hier gilt der Grundsatz, dass Handlungs- wie auch Erfolgsort zuständigkeitsbegründend sind. ${ }^{22}$

\section{2 „Oscar all'italiana“ - spürbare Interessenverletzung am Ort des angerufenen Gerichts als Merkmal des Erfolgsorts}

Dies zeigt gerade der soeben unter 2.2 dargestellte „Oscar“-Fall. Der $B G H$ bejaht dort die Zuständigkeit der deutschen Gerichte am deliktischen Erfolgsort nach Art. 7 Nr. 2 Brüssel Ia-VO mit folgender Begründung: Nach Verweis auf das so genannte Ubiquitätsprinzip wird ausgeführt, der Gerichtsstand hänge nicht davon ab, dass tatsächlich eine Verletzung des sachlichen Rechts erfolgt ist; es reiche aus, dass eine Verletzung behauptet wird und diese nicht von vornherein ausgeschlossen werden kann. ${ }^{23}$

Entscheidend war für den $B G H$ im „Oscar"-Fall, dass Verletzungsort der im Streitfall in den geltend gemachten Verstößen gegen das Markenrecht liegenden unerlaubten Handlungen auch Berlin war, weil die angegriffenen Fernsehsendungen dort im Kabelnetz und per Satellit empfangen wurden. ${ }^{24}$ Offenbleiben könne, ob sich die Sendungen bestimmungsgemäß auch an Fernsehzuschauer in Deutschland richteten. ${ }^{25}$ Im Übrigen war das hier sogar der Fall. Denn die Satellitenausstrahlung der italienischen Fernsehprogramme durch die Beklagte war darauf gerichtet, das Verständnis und die Kenntnis der italienischen Kultur und Sprache in der Welt zu fördern. Im Ergebnis erreicht der $B G H$ eine gewisse Begrenzung des potenziell an jedem Empfangs- oder Abrufort denkbaren prozessualen Erfolgsorts, wenn auch mit dem unscharfen Kriterium der Interessenverletzung am Ort des angerufenen Gerichts. ${ }^{26}$

\footnotetext{
${ }^{22}$ EuGH, GRUR 2012, 654 - Wintersteiger/Products 4U, m. Anm. Dietze, EuZW 2012, 513; zu deren Lokalisierung im Falle einer Markenrechtsverletzung s. etwa D. Paulus in Paulus/Peiffer/ Peiffer (o.Fn. 3), Art. 7 Brüssel Ia-VO Rn. 196, 204; die gleiche Zuständigkeitsregel findet sich in EuGH, GRUR 2014, 806 Rn. 46 ff. - Coty Germany/First Note, und - zum Urheberrecht - in EuGH, GRUR 2014, 100 - Pinckney/Mediatech, sowie in EuGH, GRUR 2015, 296 - Hejduk/ EnergieAgentur.

${ }^{23}$ BGH, GRUR 2012, 621 Rn. 18 - OSCAR.

${ }^{24}$ BGH, GRUR 2012, 621 Rn. 19 - OSCAR.

${ }^{25}$ BGH, GRUR 2012, 621 Rn. 21 - OSCAR; nachfolgend BGH, GRUR 2018, 935 Rn. 18 - goFit.

${ }^{26}$ Peifer, IPRax 2013, 228 (229) mit Verw. auf BGH, GRUR 2012, 621 Rn. 21 - OSCAR.
} 


\subsection{Exkurs: Geschäftsschädigende Persönlichkeitsverletzungen durch cyber mobbing - der Fall „Bolagsupplysningen“"}

\subsubsection{Lokalisierung möglicher Handlungs- und Erfolgsorte}

Den größeren systematischen Zusammenhang - und die Gefahr unzulässiger Verallgemeinerungen - verdeutlicht ein Seitenblick auf die Rolle des forum delicti commissi bei Persönlichkeitsverletzungen. Bei Verletzung von Persönlichkeitsrechten natürlicher Personen durch Website-Inhalte sind nach dem ,eDate Advertising“-Urteil des $E u G H$ jeweils zuständig: ${ }^{27}$

- die Gerichte des Mitgliedstaats, in dem der Urheber der Website-Inhalte niedergelassen ist (Handlungsort);

- die Gerichte des Mitgliedstaats, in dem der Verletzte den Mittelpunkt seiner Interessen hat (Erfolgsort 1); ${ }^{28}$

- die Gerichte des Mitgliedstaats, in dessen Hoheitsgebiet ein im Internet veröffentlichter Inhalt zugänglich ist oder war (Erfolgsort 2), mit Beschränkung der Kognitionsbefugnis auf den dort anteilig eingetretenen Schaden (Mosaikbetrachtung).

In der Entscheidung „Bolagsupplysningen“ vom 17.10.2017 ${ }^{29}$ ging es um die Zuständigkeit bei Persönlichkeitsverletzungen einer juristischen Person über das Internet, das heißt bei wirtschaftlich relevanten Rufschädigungen. ${ }^{30}$ Nicht ganz ohne Ironie fasste Generalanwalt Bobeck den zugrunde liegenden Sachverhalt so zusammen: ${ }^{31}$
„Eine in Schweden tätige estnische Gesellschaft wurde wegen angeblich fragwürdiger Ge- schäftspraktiken in eine schwarze Liste auf der Website einer schwedischen Arbeitgeber- vereinigung aufgenommen. Wie in der Ära der anonymen Internet-Tapferkeit, die allgemein für höflichen Stil, scharfen Verstand und Mäßigung bekannt ist, nicht anders zu erwarten, zog die Website eine Reihe feindseliger Kommentare ihrer Leser an. Die estnische Gesell- schaft erhob bei den estnischen Gerichten Klage gegen die schwedische Vereinigung, mit

\footnotetext{
${ }^{27}$ EuGH, GRUR 2012, 300 Ls. 1, Rn. 37 ff., 52- eDate Advertising u. Martinez, und ECLI:EU:C:2010:656 = BeckRS 2012, 80069, m. Bespr. von W.-H. Roth, IPRax 2013, 215; s. dazu etwa D. Paulus in Paulus/Peiffer/Peiffer (o.Fn. 3), Art. 7 Brüssel Ia-VO Rn. 196 f., 205 f.

${ }^{28}$ BGH, GRUR 2012, 850 - www.rainbow.at (Sedlmayr-Mord).

${ }^{29}$ EuGH, GRUR 2018, 108 - Bolagsupplysningen ua/Svensk Handel; s. dazu den Praxishinweis von Stolz, GRUR-Prax 2017, 566; Anm.: Mankowski, LMK 2017, 400139; Mann, AfP 2017, 491; Stadler, JZ 2018, 91; Ettig, K\&R 2018, 30; dazu ferner Mansel/Thorn/Wagner, IPRax 2018, 121 (135f.); Sujecki, EWS 2018, 72 (75).

${ }^{30} \mathrm{Im}$ deutschen Recht kommt hier eine Kreditgefährdung in Betracht ( $824 \mathrm{BGB}$ ), ferner unter $\S 823$ I BGB eine Verletzung des Allgemeinen Persönlichkeitsrechts oder des Rechts am eingerichteten und ausgeübten Gewerbebetrieb.

${ }^{31}$ Schlussanträge des GA Bobek, Schlussanträge v. 13.07.2017 - C-194/17, BeckRS 2017, 116694

Rn. 1 f. - Bolagsupplysningen ua/Svensk Handel.
} 
der sie rügte, dass sie durch die Veröffentlichung der Angaben in ihrer Ehre, ihrem Ansehen und ihrem guten Ruf beeinträchtigt worden sei. Sie beantragte, der schwedischen Vereinigung im Wege der Anordnung aufzugeben, die Angaben richtigzustellen und die Kommentare von ihrer Website zu entfernen. Außerdem beantragte sie Ersatz des Schadens, den sie als Folge der im Internet veröffentlichten Angaben und Kommentare erlitten haben soll.“

\title{
3.3.2 Interessenmittelpunkt als qualifizierter Erfolgsort (Erfolgsort 1; einheitlicher Erfolgsort)
}

\author{
Übernahme der „eDate Advertising“-Doktrin
}

Das Urteil „Bolagsupplysningen“ betrifft hauptsächlich das zweite in der „eDate Advertising"-Entscheidung genannte Zuständigkeitskriterium, und zwar den Mittelpunkt der Interessen des Verletzten. ${ }^{32}$ Insofern kann man von einem ,qualifizierten Erfolgsort" sprechen: Wegen seiner Verbundenheit mit dem potenziellen Opfer sind die Gerichte dieses Staats für die Geltendmachung des Gesamtschadens besonders geeignet. ${ }^{33}$

Der $E u G H$ hatte zu entscheiden, ob eine juristische Person für eine Klage aus Persönlichkeitsrechtsverletzung das forum delicti commissi in dem Mitgliedstaat in Anspruch nehmen kann, in dem sich der Mittelpunkt ihrer Interessen befindet und, wenn ja, welche Kriterien und Umstände zur Bestimmung des Mittelpunkts der Interessen heranzuziehen sind. Der $E u G H$ bestätigte im Ausgangspunkt sein Urteil im Fall „eDate Advertising“, wo neben dem Handlungs- und Erfolgsort auch der Ort des Mittelpunkts der Interessen einer natürlichen Person als Ort des schädigenden Ereignisses eingestuft wurde. ${ }^{34}$ Im Fall „Bolagsupplysningen“ hat der $E u G H$ diese Aussage auf juristische Personen erstreckt. Jeder Kläger könne im Falle einer Persönlichkeitsrechtsverletzung bei den Gerichten des Orts des Mittelpunkts seiner Interessen den gesamten Schaden einklagen. Dies sei im Interesse einer geordneten Rechtspflege gerechtfertigt und diene dem besonderen Schutz des Klägers, weil sich an dessen Interessenmittelpunkt die Persönlichkeitsverletzung ,am spürbarsten verwirklicht"

\footnotetext{
${ }^{32}$ EuGH, GRUR 2018, 108 Rn. 22-44 - Bolagsupplysningen ua/Svensk Handel.

${ }^{33}$ Vgl. W.-H. Roth, IPRax 2013, 215 (220); zum Urteil „eDate Advertising“; andere Begrifflichkeit bei Mansel/Thorn/Wagner, IPRax 2018, 212 (136): ,einheitlicher Erfolgsort“ im Gegensatz zum „Teilerfolgsort“, an dem lediglich die Abrufbarkeit der verletzenden Internetinhalte gegeben ist (das dritte Zuständigkeitskriterium nach dem Urteil „eDate Advertising“); krit. zum Mittelpunkt der klägerischen Interessen als Erfolgsort Mankowski in Magnus/Mankowski, Brussels Ibis Regulation, 2016, Art. 7 Rn. 365 ff.

${ }^{34}$ EuGH, GRUR 2012, 300 Rn. 48 ff. - eDate Advertising u. Martinez; hierzu Sujecki, K\&R 2011, 315; Heinze, EuZW 2011, 947; Picht, GRUR Int 2013, 19.

${ }^{35}$ EuGH, GRUR 2018, 108 Rn. 33, 42 - Bolagsupplysningen ua/Svensk Handel; abl. Mankowski, LMK 2017, 400139; dazu Mansel/Thorn/Wagner, IPRax 2018, 121 (136).
} 


\section{Lokalisierung des Interessenmittelpunkts der juristischen Person}

Für die Lokalisierung des Interessenmittelpunkts von natürlichen Personen hatte der $E u G H$ im Urteil „eDate Advertising“ den im europäischen internationalen Privat- und Prozessrecht vielfach verwendeten gewöhnlichen Aufenthalt der Person herangezogen. ${ }^{36}$ Dies entspricht auch der Regelung in Art. 3 I UAbs. 4 EuInsVO zum Mittelpunkt der hauptsächlichen Interessen einer natürlichen Person, welche nicht gewerblich tätig ist. ${ }^{37}$ In Bezug auf die Kriterien und Umstände zur Bestimmung des Mittelpunkts der Interessen bei einer juristischen Person zieht der EuGH nun freilich keine Parallele zum internationalen Insolvenzrecht. Es gibt daher keine diesbezügliche Vermutung zugunsten des Satzungssitzes wie nach Art. 3 I UAbs. 2 S. 1 EuInsVO. ${ }^{38}$ Vielmehr hat der $E u G H$ - etwas diffus - festgestellt, dass der Ort des Mittelpunkts der Interessen einer juristischen Person dort liegen müsse, wo deren geschäftliches Ansehen am gefestigtsten ist. ${ }^{39}$ Er sei daher anhand des Ortes zu bestimmen, an dem die juristische Person den wesentlichen Teil ihrer wirtschaftlichen Tätigkeit ausübt. Dieses könne zwar einerseits der Ort ihres satzungsmäßigen Sitzes sein, allerdings sei dies nicht zwingend, da sich die wirtschaftliche Tätigkeit auch auf einen anderen Mitgliedstaat erstrecken kann. ${ }^{40}$ Es spricht einiges dafür, hier im Wege der rechtsaktübergreifenden Auslegung auf die umfangreiche Rechtsprechung und Literatur zur Lokalisierung des insolvenzrechtlichen Centre of Main Interests (COMI) zurückzugreifen. ${ }^{41}$

\section{„Abrufstaat“ als Teilerfolgsort (Erfolgsort 2) mit beschränkter Kognitionsbefugnis}

Das Urteil äußert sich auch noch knapp zum dritten in der ,eDate Advertising“-Entscheidung genannten Zuständigkeitskriterium, dem „Erfolgsort 2“, an dem der streitige Internetinhalt lediglich zugänglich - das heißt abrufbar - ist. ${ }^{42}$ Hier geht es im Kern um die Ubiquität des Internets, aus der keine Ubiquität der Gerichtspflich-

\footnotetext{
${ }^{36}$ EuGH, GRUR 2012, 300 Rn. 49 - eDate Advertising u. Martinez; dazu W.-H. Roth, IPRax 2013, 215 (217 f.); rudimentär auch in der Schlussentscheidung: BGH, IPRax 2013, 252 Rn. 18: „Hier wohnt [der Kläger] und ist sozial und familiär eingebunden. [...] Hier wirkt sich eine Verletzung seines Achtungsanspruchs aus.“

${ }^{37}$ „Bei allen anderen natürlichen Personen wird bis zum Beweis des Gegenteils vermutet, dass der Mittelpunkt ihrer hauptsächlichen Interessen der Ort ihres gewöhnlichen Aufenthalts ist."“; dazu MüKoBGB/Kindler (o. Fn. 12), Art. 3 EuInsVO Rn. 39 ff.

${ }^{38}$ Mansel/Thorn/Wagner, IPRax 2018, 121 (136).

${ }^{39}$ EuGH, GRUR 2018, 108 Rn. 41 - Bolagsupplysningen ua/Svensk Handel.

${ }^{40}$ EuGH, GRUR 2018, 108 Rn. 41 - Bolagsupplysningen ua/Svensk Handel; Sujecki, EWS 2018, 72 (76).

${ }^{41}$ Wie im Text auch Hau, GRUR 2018, 163 (164); zum COMI MüKoBGB/Kindler (o. Fn. 12), Art. 3 EuInsVO Rn. 14 ff.

${ }^{42}$ EuGH, GRUR 2018, 108 Rn. 45-49 - Bolagsupplysningen ua/Svensk Handel.
} 
tigkeit folgen darf. ${ }^{43}$ Fraglich war, ob eine Person eine Klage auf Richtigstellung falscher Angaben aufgrund der freien Zugänglichkeit des Internets vor den Gerichten jedes Mitgliedstaats erheben könne, in dessen Hoheitsgebiet die Informationen zugänglich waren. Der $E u G H$ verneinte diese Frage überzeugend, da ein auf die Richtigstellung persönlichkeitsverletzender Angaben und die Entfernung dieser Inhalte gerichteter Antrag einheitlich und untrennbar ist. Bei mehrfacher internationaler Zuständigkeit bestünde die Gefahr eines forum shopping und einander widersprechender Entscheidungen. Daher ist die bloße Abrufbarkeit einer Internetseite nicht ausreichend für die Begründung der Zuständigkeit. Diese Regel gilt laut $E u G H$ auch für bloße Unterlassungsansprüche. Für diese Ansprüche sind die Gerichte zuständig, die auch für den Ersatz des gesamten Schadens zuständig sind. ${ }^{44}$

Der im Urteil „eDate Advertising“ erwähnte Erfolgsort 2 (Teilerfolgsort) ${ }^{45} \mathrm{im}$ Abrufstaat begründet daher für juristische Personen einen Gerichtsstand nur für Schadensersatzklagen - nicht: Klagen auf Richtigstellung - und auch insoweit nur für den in dem betreffenden Staat eingetretenen Teilschaden (Mosaikbetrachtung).

\section{4 „Wintersteiger": Deliktsort bei der Markenverletzung}

\subsubsection{Fallsituation und Problemlage}

Um das Ubiquitätsprinzip ging es auch in dem vom EuGH 2012 erlassenen Urteil im Fall „Wintersteiger/Products $4 U^{4} .{ }^{46}$ Danach ergeben sich signifikante Unterschiede zwischen der Lokalisierung des Deliktsorts bei der Markenverletzung und bei der soeben behandelten Persönlichkeitsrechtsverletzung.

Zum Sachverhalt: Ein Werbender verwendete auf der Website einer Suchmaschine, die unter der Top-Level-Domain eines anderen Mitgliedstaats betrieben wird, ein mit der Marke identisches Schlüsselwort. Zuständig für die Entscheidung über Schadensersatz- und Unterlassungsansprüchen des Markenrechtsinhabers sind laut $\mathrm{EuGH}$ sowohl die Gerichte des Mitgliedstaats, in dem die Marke eingetragen ist (deliktischer Erfolgsort) wie auch die Gerichte desjenigen Mitgliedstaats, in dem der Werbende niedergelassen ist (deliktischer Handlungsort).

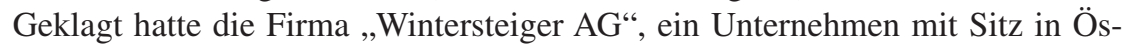
terreich, das in der Herstellung und im Vertrieb von Ski- und SnowboardServicemaschinen nebst Ersatzteilen und Zubehör tätig ist. Die Klägerin ist Inhaberin der österreichischen Marke „Wintersteiger“. Das Unternehmen „Products 4U“ mit Sitz in Deutschland entwickelt und vertreibt ebenfalls Ski- und Snowboard-

\footnotetext{
${ }^{43}$ Sujecki, EWS 2018, 72 (76).

${ }^{44}$ EuGH, GRUR 2018, 108 Rn. 47 f. - Bolagsupplysningen ua/Svensk Handel.

${ }^{45}$ Mansel/Thorn/Wagner, IPRax 2018, 212 (136); EuGH, GRUR 2012, 300 Rn. 42 - eDate Advertising u. Martinez.

${ }^{46}$ EuGH, GRUR 2012, 654 - Wintersteiger/Products 4U, mit Anm. Dietze, EuZW 2012, 514; dazu v. Hein, LMK 2012, 333913; Mansel/Thorn/Wagner, IPRax 2013, 1 (16).
} 
Servicemaschinen. Darüber hinaus verkauft Products 4U Zubehör für Maschinen anderer Hersteller, insbesondere für jene von Wintersteiger. Dieses Zubehör, das Products 4U als „Wintersteiger- Zubehör“ bezeichnet, stammt weder aus der Herstellung von Wintersteiger, noch ist es von ihr autorisiert. Wie Wintersteiger ist Products 4U weltweit tätig und vertreibt ihre Waren insbesondere auch in Österreich. Products $4 \mathrm{U}$ hatte in dem von dem Anbieter des Internetreferenzierungsdienstes Google entwickelten Anzeigensystem „Wintersteiger“ als Schlüsselwort (,AdWord“) gebucht. Nach dieser Buchung, die auf die deutsche länderspezifische Toplevel-Domain von Google, das heißt die Website „google.de“, beschränkt war, erschien bei Eingabe von „Wintersteiger“ in die Suchmaschine dieses Referenzierungsdienstes als erstes Suchergebnis ein Link zur Website von Wintersteiger. Außerdem erschien bei Eingabe dieses Suchbegriffs jedoch am rechten Bildschirmrand unter der Überschrift „Anzeige“ eine Werbeanzeige von Products 4U. Darüber, ob dies eine Verletzung der Marke „Wintersteiger“ darstellt, kann laut EuGH vor österreichischen Gerichten (da dort die Marke Wintersteiger eingetragen ist) oder vor deutschen Gerichten (Niederlassung des angeblichen Markenrechtsverletzers Products $4 \mathrm{U})$ prozessiert werden. ${ }^{47}$

\subsubsection{Beschränkung der Erfolgsortzuständigkeit auf den Staat der Markeneintragung}

Im Hinblick auf den deliktischen Erfolgsort stellt sich allerdings die Frage, ob und inwiefern dieses Merkmal im Lichte des Territorialitätsprinzips, das für Immaterialgüterrechte prägend ist (oben 2.1), einer gewissen Einschränkung bedarf. Dafür spricht jedenfalls prima facie das Primat der Wohnsitzzuständigkeit (Art. 4 Brüssel Ia-VO) und der Auslegungszusammenhang mit der Rom II-VO und deren Art. 8. ${ }^{48}$

Den Erfolgsort lokalisierte der EuGH im Fall „Wintersteiger“ daher in Österreich, weil dort die streitgegenständliche nationale Marke eingetragen war und die dortigen Gerichte folglich am besten in der Lage seien zu beurteilen, ob tatsächlich eine Verletzung des geschützten Rechts vorliege. ${ }^{49}$ Den Umstand, dass Products 4U das Schlüsselwort „Wintersteiger“ nur für die deutsche und nicht für die österreichische Seite des Internet-Suchdienstes Google gebucht hatte, hält der EuGH kompetenzrechtlich nicht für ausschlaggebend; ob dieser Faktor eine Markenrechtsverletzung im Ergebnis ausschließe, betreffe nämlich die Begründetheit und nicht die Zulässigkeit der Klage. ${ }^{50}$ Damit liegt der $E u G H$ auf einer Linie mit dem $B G H$, der es im Fall „Hotel Maritime“ für die Zuständigkeit genügen ließ, wenn eine Verletzung behauptet werde und diese nicht von vornherein ausgeschlossen werden

\footnotetext{
${ }^{47}$ Anwendbares Recht: Art. 8 Rom II-VO.

${ }^{48} \mathrm{Vgl}$. Erwägungsgrund 7 zur Rom II-VO; dazu oben bei Fn. 8.

${ }^{49}$ EuGH, GRUR 2012, 654 - Wintersteiger/Products 4U; nachfolgend BGH, GRUR 2018, 935

Rn. 17 - goFit.

${ }^{50}$ EuGH, GRUR 2012, 654 - Wintersteiger/Products 4U.
} 
könne. ${ }^{51}$ Hier besteht ein Unterschied zu urheberrechtlichen Streitigkeiten. Im Markenrecht würde der $B G H$ wohl einen schlüssigen Vortrag der Verletzung verlangen und einen solchen verneinen, wenn eine wirtschaftliche Auswirkung auf das Inland (und damit nach „Hotel Maritime“ und „OSCAR“ eine materiell-rechtliche Verletzung) ausgeschlossen wäre. Diese Einschränkung lehnt der $E u G H$ im Urheberrecht $a b .{ }^{52}$

\subsubsection{Kein qualifizierter Erfolgsort (Erfolgsort 1; einheitlicher Erfolgsort) am Interessenmittelpunkt des Schutzrechtsinhabers}

Eine deutliche Abgrenzung nimmt der EuGH in Bezug auf seine oben unter 3.3 dargestellte Rechtsprechung zu Persönlichkeitsrechtsverletzungen vor: Während insoweit der Kläger an seinem gewöhnlichen Aufenthalt als „,Mittelpunkt seiner Interessen" auch bei Streudelikten - das heißt bei Verletzungen des Persönlichkeitsrechts in mehreren Staaten - den gesamten Schaden einklagen kann, ${ }^{53}$ ist die Kognitionsbefugnis der Gerichte am Erfolgsort im Immaterialgüterrecht auf die Verletzung des gerade dort eingetragenen Schutzrechts beschränkt. ${ }^{54}$ Der EuGH begründet dies überzeugend mit Verweis auf das immaterialgüterrechtliche Territorialitätsprinzip ${ }^{55}$ und stellt damit zugleich eine Wertungsparallele zum kollisionsrechtlichen Schutzlandprinzip (Art. 8 Rom II-VO; oben 2.1) her. ${ }^{56}$

\subsubsection{Beschränkung der Handlungsortzuständigkeit}

Nicht restlos geklärt ist, ob es bei Immaterialgüterrechtsverletzungen ontologisch überhaupt einen vom Erfolgsort zu trennenden Handlungsort gibt. Nach der in der IP-Literatur wohl herrschenden Auffassung fallen der Handlungs- und der Erfolgsort notwendig am Ort der Verletzung zusammen, da außerhalb des kollisionsrechtlich maßgeblichen Schutzlands keine Verletzungshandlung vorgenommen werden könne. ${ }^{57}$ Dieser eher begrifflich-logischen Betrachtungsweise will der EuGH im

\footnotetext{
${ }^{51}$ BGH, GRUR 2005, 431 - Hotel Maritime, mit Anm. Berlit, LMK 2005, 78.

${ }^{52}$ EuGH, GRUR 2014, 100 - Pinckney; GRUR 2015, 296 - Hejduk Mediatech; krit. wegen der daraus folgenden potenziellen Uferlosigkeit der Zuständigkeit Picht/Kopp, GRUR Int 2016, 232.

${ }^{53} \mathrm{EuGH}$, GRUR 2012, 300 Rn. 49, 52 - eDate Advertising u. Martinez, mit Anm. Leible, LMK 2012, 329468.

${ }^{54} \mathrm{EuGH}$, GRUR 2012, 654 Rn. 25, 28 - Wintersteiger/Products 4U. Nur im Urheberrecht - anders als im Markenrecht - besteht dennoch potenziell eine Vielzahl von Zuständigkeiten, weil das Urheberrecht formlos in allen Mitgliedstaaten der Berner Übereinkunft entsteht.

${ }^{55}$ EuGH, GRUR 2012, 654 Rn. 25 - Wintersteiger/Products 4U; Mansel/Thorn/Wagner, IPRax 2013, 1 (16).

${ }^{56}$ Zur Herleitung der Schutzlandanknüpfung aus dem Territorialitätsgrundsatz MüKoBGB/Drexl (o. Fn. 12), Internationales Immaterialgüterrecht Rn. 7 ff.; ferner BGH, GRUR 2012, 621 Rn. 34 - OSCAR.

${ }^{57}$ Lehmann/Stieper, JZ 2012, 1016; Peifer, IPRax 2013, 228; ferner v. Hein, LMK 2012, 333913
} 
Urteil „Wintersteiger“ nicht folgen: ${ }^{58}$ Namentlich im Hinblick auf die Beweisnähe des Forums könne auch außerhalb des Schutzgebiets einer nationalen Marke die Eröffnung des europäischen Deliktsgerichtsstands gerechtfertigt sein. ${ }^{59}$

Als zuständigkeitsrechtlich relevante Verletzungshandlung bei der Verwendung einer Marke als Schlüsselwort betrachtet der $E u G H$ weder das Erscheinen der Werbung auf dem Monitor der Nutzer noch die Einspeisung der Daten vom Server des Betreibers der Suchmaschine: Der erstgenannte Vorgang stelle kein Verhalten des Werbenden dar; ${ }^{60}$ der Serverstandort sei nicht vorhersehbar. ${ }^{61}$ Als Handlungsort betrachtet der $E u G H$ vielmehr den Ort der Niederlassung des Werbenden, an dem über das Auslösen des technischen Anzeigevorgangs entschieden werde, ${ }^{62}$ im vorliegenden Fall also die Niederlassung der deutschen Beklagten. ${ }^{63}$ Die darin liegende Parallele zur Lokalisierung des Handlungsorts in den cyber mobbing-Fällen - oben 3.3.1 - fällt auf, wird vom $E u G H$ aber nicht angesprochen.

Im Hinblick auf die Vorhersehbarkeit der Gerichtspflichtigkeit potenzieller Verletzer ist diese Einschränkung des Handlungsortsbegriffs jedenfalls zu begrüßen. ${ }^{64}$ Restzweifel bleiben freilich, wenn man etwa den Fall „Knoll“" vergleichend heranzieht: Dort wurden Designermöbel aus Italien im Internet gegenüber Deutschen beworben und nur in Deutschland bestand Urheberschutz. ${ }^{65}$ Hier war die Verletzungshandlung grenzüberschreitend; insofern könnte man in „Wintersteiger“ durchaus auch eine Verletzungshandlung in Österreich wegen der dortigen Abrufbarkeit der Website annehmen.

\subsubsection{Bewertung: Stärkung des Prinzips actor sequitur forum rei}

In der Gesamtschau erschwert der $E u G H$ dem Markeninhaber bei grenzüberschreitenden Rechtsverletzungen die Rechtsdurchsetzung vor heimischen Gerichten: Zwar kann im nach Art. 8 Rom II-VO maßgeblichen Schutzland (Österreich) als Erfolgsort geklagt werden, doch dürfte es im materiellen Recht an einer relevanten

mit Verw. auf die CLIP-Principles, abgedr. bei Basedow/Kono/Metzger, Intellectual Property in the Global Arena, 2010, Annex II.

${ }^{58} \mathrm{EuGH}$, GRUR 2012, 654 Rn. 20 - Wintersteiger/Products 4U.

${ }^{59} \mathrm{EuGH}$, GRUR 2012, 654 Rn. 32 - Wintersteiger/Products 4U.

${ }^{60} \mathrm{EuGH}$, GRUR 2012, 654 Rn. 34 -Wintersteiger/Products 4U.

${ }^{61}$ EuGH, GRUR 2012, 654 Rn. 36 - Wintersteiger/Products 4U.

${ }^{62}$ EuGH, GRUR 2012, 654 Rn. 37 - Wintersteiger/Products 4U.

${ }^{63}$ Der BGH möchte - bei den Unionsschutzrechten - im Anschluss an ein kollisionsrechtliches EuGH-Urteil (EuGH, GRUR 2017, 1120 - Nintendo/Big Ben) den Handlungsort in einer diffusen Gesamtbetrachtung bestimmen: BGH, GRUR 2018, 84 - Parfummarken; dazu mit Recht krit. Kur, GRUR 2018, 358.

${ }^{64}$ Ebenso W.-H. Roth, IPRax 2013, 215 (217), der die Parallele zum Urteil „Shevill“ betont (Niederlassung des Herausgebers einer ehrverletzenden Veröffentlichung als alleiniger Handlungsort), vgl. EuGH, EuZW 1995, 248 Rn. 24, 32 - Shevill, mit Bespr. von Kreuzer/Klötgen, IPRax 1997, 90; dazu auch $P$. Huber, ZEuP 1996, 300.

${ }^{65} \mathrm{EuGH}$, GRUR 2015, 665 - Dimensione ua/Knoll [Marcel-Breuer-Möbel]. 
Verletzungshandlung fehlen, wenn man insoweit - mit dem $E u G H$ - auf die Vorgänge an der im Ausland (Deutschland) befindlichen Niederlassung des angeblichen Markenverletzers abstellt. ${ }^{66}$ Denn laut $E u G H$ ist ja die maßgebliche Handlung so zuzuschneiden, dass sie regelmäßig am allgemeinen Gerichtsstand des angeblichen Verletzers stattfindet.

Unter Berufung auf den Handlungsort kommt der Markeninhaber nicht zu einem Klägergerichtsstand: Lokalisiert man nämlich den Handlungsort an der ausländischen Niederlassung des angeblichen Verletzers, so geht der allgemeine Gerichtsstand nach Art. 4 I Brüssel Ia-VO ohnehin dem Deliktsgerichtsstand nach Art. 7 Nr. 2 Brüssel Ia-VO vor. ${ }^{67}$ Hinzu kommt der fehlende Gleichlauf zum anwendbaren Recht. Eine Klageerhebung am allgemeinen Gerichtsstand außerhalb des Staats der lex loci protectionis dürfte nur selten zweckmäßig sein, müsste doch das angerufene Gericht in diesem Fall das ihm fremde Recht des Schutzlandes kostspielig und zeitraubend ermitteln ( $\$ 293 \mathrm{ZPO})$.

\section{Abgrenzung zur ausschließlichen Zuständigkeit bei Streitigkeiten über die Markeninhaberschaft (Art. 24 Nr. 4 Brüssel Ia-VO) - ,Hanssen/Knipping“6}

\subsection{Sachlicher Geltungsbereich des Gerichtsstands der gewerblichen Schutzrechte}

Als Wahlgerichtsstand tritt der allgemeine europäische Deliktsgerichtsstand - Art. 7 Nr. 2 Brüssel Ia-VO - hinter die in der Brüssel Ia-VO normierten ausschließlichen Zuständigkeiten zurück. Dazu zählt auf dem Gebiet der Immaterialgüterrechte Art. 24 Nr. 4 S. 1 Brüssel Ia-VO, wonach für bestimmte Streitigkeiten die ausschließliche internationale Zuständigkeit der Gerichte im Hinterlegungs- oder Registrierungsstaat besteht. ${ }^{68}$ Dabei geht es um Verfahren, welche die Eintragung oder die Gültigkeit von Patenten, Marken, Mustern und Modellen sowie ähnlicher Rechte, die einer Hinterlegung oder Registrierung bedürfen, zum Gegenstand haben. Die ausschließliche Zuständigkeit soll der besonderen Sachnähe der Gerichte

\footnotetext{
${ }^{66}$ Vgl. nochmals EuGH, GRUR 2012, 654 Rn. 37 - Wintersteiger/Products 4U (,Vielmehr ist der Ort der Niederlassung des Werbenden als der Ort anzusehen, an dem über das Auslösen des technischen Anzeigevorgangs entschieden wird, da es sich sowohl für den Kl. als auch für den Bekl. um einen feststehenden und feststellbaren Ort handelt, der daher geeignet ist, die Beweiserhebung und die Gestaltung des Prozesses zu erleichtern.“; gleichsinnig v. Hein, LMK 2012, 333913.

${ }^{67}$ Treffend Mansel/Thorn/Wagner, IPRax 2013, 1 (16): „Allerdings marginalisiert diese Bestimmung des Handlungsorts das Wahlrecht des Geschädigten aus Art. 5 Nr. 3 EuGVVO [nunmehr Art. 7 Nr. 2 Brüssel Ia-VO]...“.

${ }^{68}$ Kropholler/v. Hein (o. Fn. 8), Art. 5 Brüssel I-VO Rn. 74; D. Paulus in Paulus/Peiffer/Peiffer (o.Fn. 3), Art. 24 Brüssel Ia-VO Rn. 108 ff.
} 
im Hinterlegungs- oder Registrierungsstaat Rechnung tragen ${ }^{69}$ und einen Gleichlauf von Zuständigkeit und materiellem Recht sichern. ${ }^{70}$

Der Kreis der erfassten Klagearten ist - wie bei allen ausschließlichen Zuständigkeiten - eng zu ziehen. ${ }^{71}$ Daher sind die allgemeinen Gerichtsstände der Verordnung - und damit auch das forum delicti commissi - für alle Klagen wegen einer Verletzung des gewerblichen Schutzrechts eröffnet, ${ }^{72}$ einschließlich der Unterlassungsklagen. ${ }^{73}$ Nicht erfasst werden sollen demgemäß Vindikationsklagen nach § 8 PatG und Eintragungsbewilligungsklagen nach $\S 44$ MarkenG. $^{74}$ Auch Streitigkeiten über vertraglich begründete Ansprüche auf Einräumung von Schutzrechten begründen keine ausschließliche internationale Zuständigkeit. ${ }^{75}$ Dabei darf nach der umstrittenen, seit der Neufassung der Brüssel Ia-VO sogar in Gesetzesform (,unabhängig davon, ob die Frage im Wege der Klage oder der Einrede aufgeworfen wird") gegossenen „GAT“-Entscheidung des $E u G H$ jedoch im Verletzungsprozess nicht inzident über den streitigen Bestand des Schutzrechts entschieden werden, da insoweit die ausschließliche Zuständigkeit nach Art. 24 Nr. 4 Brüssel Ia-VO vorgeht. ${ }^{76}$ Der $\mathrm{EuGH}$ betont in diesem Zusammenhang besonders den territorialen Charakter der Schutzrechte (oben 2.1) und die Notwendigkeit einer Entscheidung im „Ursprungsland“, welches auch über die Tragweite einer Nichtigerklärung entscheiden müsse (nur inter partes oder erga omnes?). ${ }^{77}$

${ }^{69}$ D. Paulus in Paulus/Peiffer/Peiffer (o.Fn. 3), Art. 24 Brüssel Ia-VO Rn. 111; zum Patentrecht EuGH, GRUR Int 1984, 693 - Duijnstee, mit Anm. Stauder; BGH, GRUR 2007, 884 - Cambridge Institute, mit Bespr. von Reichardt, IPRax 2008, $330 \mathrm{ff}$.

${ }^{70}$ Adolphsen, IPRax 2007, 15 (17); Stadler in Musielak/Voit (o. Fn. 3), Art. 24 Brüssel Ia-VO Rn. 9. ${ }^{71}$ EuGH, GRUR Int 1984, 693 - Duijnstee, mit Anm. Stauder; BGH, GRUR 2007, 884 - Cambridge Institute; D. Paulus in Paulus/Peiffer/Peiffer (o. Fn. 3), Art. 24 Brüssel Ia-VO Rn. 116.

${ }^{72}$ Bericht von P. Jenard zu dem Übereinkommen v. 27.09.1968 über die gerichtliche Zuständigkeit und die Vollstreckung gerichtlicher Entscheidungen in Zivil- und Handelssachen, ABl. EG 1979 Nr. C 59 v. 05.03.1979, 34; D. Paulus in Paulus/Peiffer/Peiffer (o. Fn. 3), Art. 24 Brüssel Ia-VO Rn. 121; Adolphsen, IPRax 2007, 15 (17); bestätigt der Sache nach von EuGH, GRUR 2007, 49 - GAT.

${ }^{73}$ Die Zuständigkeit für Patentverletzungsklagen kann aus Art. 4 I, 7 Nr. 2 oder aus Art. 8 Nr. 1 Brüssel Ia-VO herzuleiten sein.

${ }^{74}$ D. Paulus, Paulus/Peiffer/Peiffer (o. Fn. 3), Art. 24 Brüssel Ia-VO Rn. 120.

${ }^{75}$ Thünken, GRUR-Prax 2017, 483; Kropholler/von Hein (o. Fn. 8), Art. 5 Brüssel I-VO Rn. 48.

${ }^{76} \mathrm{EuGH}$, GRUR 2007, 49 - GAT, und GRUR 2007, 47 - Roche; krit. hierzu Kropholler/von Hein (o.Fn. 8), Art. 5 Brüssel I-VO Rn. 50; D. Paulus in Paulus/Peiffer/Peiffer (o. Fn. 3), Art. 24 Brüssel Ia-VO Rn. 122 ff.; Hess, EuZPR, 2010, § 6 Rn. 123 f. (der plakativ von einem „Supertorpedeo“ spricht: Der Beklagte kann mit der Behauptung, das Patent sei unwirksam, den Prozess am Verletzungsort blockieren); Adolphsen, IPRax 2007, 15; Reichardt, GRUR Int 2008, 574; Gottschalk, JZ 2007, 299; s. auch Knaak, GRUR Int 2007, 386; Luginbühl/Stauder, GRUR Int 2014, 885 (890).

${ }^{77}$ Stadler in Musielak/Voit (o. Fn. 3), Art. 24 Brüssel Ia-VO Rn. 9. 


\subsection{Prätendentenstreit-Abgrenzung nach dem Grad der „Registernähe“?}

Nicht von Art. 24 Nr. 4 Brüssel Ia-VO erfasst sind grundsätzlich Streitigkeiten der Parteien darüber, wem ein gewerbliches Schutzrecht zusteht. ${ }^{78}$ Diesen Grundsatz hat der $E u G H$ jüngst im Urteil „Hanssen“ vom 05.10.2017 verdeutlicht, ${ }^{79}$ dem ein Prätendentenstreit von Markeninhabern zugrunde lag: Frau Prast-Knipping, eine in Deutschland wohnhafte Inhaberin einer Benelux-Marke, hatte deren Eintragung nach Vorlage eines Erbscheins durch Umschreibung ${ }^{80}$ erlangt. Sie wurde durch die in den Niederlanden ansässige Hanssen Beleggingen BV auf Abgabe einer Verzichtserklärung auf Markeninhaberschaft gegenüber dem Benelux-Markenamt verklagt. ${ }^{81}$ Hanssen machte ungerechtfertigte Bereicherung nach $\S 812$ BGB geltend und verlangte von Frau Prast-Knipping die Erklärung gegenüber dem BeneluxMarkenamt, dass sie hinsichtlich dieser Marke Nichtberechtigte sei und auf die Eintragung als Markeninhaberin verzichte. Nach dem Vortrag der Klägerin war die beanstandete Marke vor dem Tod des Erblassers Gegenstand mehrerer Übertragungen und hat sich zum Zeitpunkt seines Todes nicht mehr in dessen Vermögen befunden.

Gegenstand der Vorlage nach Art. 267 AEUV war die Frage, welche registernahen Rechtstreitigkeiten über die Markeninhaberschaft der ausschließlichen Zuständigkeit unterfallen. Laut $E u G H$ beschränkt sich Art. 24 Nr. 4 Brüssel Ia-VO darauf, Rechtsstreitigkeiten über die Eintragung oder die Gültigkeit eines Rechts des geistigen Eigentums denjenigen Gerichten vorzubehalten, die eine sachliche und rechtliche Nähe zum Register aufweisen; diese Gerichte seien am besten in der Lage, über Fälle zu entscheiden, in denen die Gültigkeit des Rechts und sogar das Bestehen seiner Hinterlegung oder Registrierung selbst, bestritten wird. ${ }^{82}$ Folglich sei ein Rechtsstreit, in dem weder die Eintragung der Marke als solche beanstandet noch deren Gültigkeit bestritten wird, nicht vom Zweck dieser Bestimmung erfasst. Die Frage, in wessen persönliches Vermögen ein Recht des geistigen Eigentums fällt, weise insoweit im Allgemeinen keine sachliche oder rechtliche Nähe zum Ort der Eintragung dieses Rechts auf. ${ }^{83}$

\footnotetext{
${ }^{78}$ EuGH, GRUR Int 1984, 693 - Duijnstee, mit Anm. Stauder; GRUR 2017, 1167 - Hanssen/ Prast-Knipping [knipping], mit Bespr. von Mankowski, IPRax 2018, 355; Borás/Hausmann in Simons/Hausmann, Brüssel I-VO, 2012, Rn. 69 mN zur Rspr. in den Mitgliedstaaten.

${ }^{79} \mathrm{EuGH}$, GRUR 2017, 1167 - Hanssen/Prast-Knipping [knipping]; s. hierzu Thünken, GRURPrax 2017, 483; Sujecki, EWS 2018, 72 (79); Mansel/Thorn/Wagner, IPRax 2018, 121 (137); Mankowski, IPRax 2018, 355.

${ }^{80} \mathrm{Im}$ deutschen Recht $§ 27$ I und III MarkenG.

${ }^{81} \mathrm{Vgl}$. im deutschen Recht $§ 48$ MarkenG; für Italien Art. 15 IV und V c.p.i.

${ }^{82} \mathrm{EuGH}$, GRUR 2017, 1167 Rn. 33 - Hanssen/Prast-Knipping [knipping].

${ }^{83}$ EuGH, GRUR 2017, 1167 Rn. 34 ff. - Hanssen/Prast-Knipping [knipping].
} 


\subsection{Kritik: unscharfe Abgrenzung der registernahen Prätendentenstreitigkeiten}

Damit hat der $E u G H$ den Anwendungsbereich der ausschließlichen Zuständigkeit nach Art. 24 Nr. 4 Brüssel Ia-VO auf Klagen beschränkt, die nur auf die Eintragung und Gültigkeit eines Schutzrechts gerichtet sind ${ }^{84}$ Alle weiteren Klagen - wie zum Beispiel im Ausgangsverfahren bezüglich der Inhaberschaft - werden nicht von der ausschließlichen Zuständigkeit nach Art. 24 Nr. 4 Brüssel Ia-VO erfasst. ${ }^{85}$ Die Argumentation des $E u G H$ entlang dem Wortlaut der Bestimmung wird dem Grundsatz der engen Auslegung der ausschließlichen Zuständigkeiten - oben 4.1 - gerecht, erscheint aber mit der vom $E u G H$ vertretenen Erstreckung des Art. 24 Nr. 4 Brüssel Ia-VO auf Nichtigkeitseinreden im Verletzungsprozess ${ }^{86}$ kaum vereinbar. ${ }^{87}$ Auch blieb unberücksichtigt, dass eine Zuständigkeit der Gerichte des Eintragungsstaats nach Art. 24 Nr. 3 Brüssel Ia-VO in Betracht kommen kann. ${ }^{88}$ Der EuGH beschränkt den Gerichtsstand der gewerblichen Schutzrechte auf Verfahren, die unmittelbar die Wirksamkeit oder Existenz des Schutzrechts selbst betreffen ${ }^{89}$ Die Fallgruppe umfasst Klagen - oder Widerklagen - auf Löschung oder Feststellung der Unwirksamkeit eines Schutzrechts. Sofern es hingegen im Kern um die Inhaberschaft am Schutzrecht geht, ist kein Raum für eine ausschließliche internationale Zuständigkeit der Gerichte des Hinterlegungsstaats.

\section{Ergebnisse in Thesen}

1. Der europäische Deliktsgerichtsstand am Tatort beruht auf dem Grundgedanken, dass der durch eine unerlaubte Handlung angeblich Verletzte seine Schadensersatzansprüche dort geltend machen darf, wo das behauptete Unrecht begangen wurde, sich ausgewirkt hat oder sich auszuwirken droht. Hinzukommt das Prinzip der Sach- und Beweisnähe. Als Ausnahme zum Grundsatz actor sequitur forum rei (Art. 4 Brüssel Ia-VO) ist der Deliktsgerichtsstand allerdings eng zu verstehen.

\footnotetext{
${ }^{84}$ Sujecki, EWS 2018, 72 (79).

${ }^{85}$ So Thünken, GRUR-Prax 2017, 483; Mankowski, IPRax 2018, 355 (360).

${ }^{86} \mathrm{EuGH}$, GRUR 2007, 49 - GAT; dazu oben bei Fn. 76.

${ }^{87}$ Vom Urteil „GAT“ (s. Fn. 86) hat sich das Urteil „Hanssen“ nicht distanziert: EuGH, GRUR 2017, 1167 Rn. 31, 33, 34 - Hanssen/Prast-Knipping [knipping]; unzutr. Thünken, GRUR-Prax 2017, 483 mit Verw. auf D. Paulus in Paulus/Peiffer/Peiffer (o. Fn. 3), Art. 24 Brüssel Ia-VO Rn. 122 ff. („Hinzu kommt, dass die Eintragung bzw. Gültigkeit eines Schutzrechts nicht nur als Vorfrage oder Einrede [etwa in einem Patent- oder Markenverletzungsprozess], sondern als Hauptfrage in einem Rechtsstreit Relevanz erlangen muss, damit der ausschließliche internationale Gerichtsstand greift ...").

${ }^{88}$ Dafür mit guten Gründen Mankowski, IPRax 2018, 355 (357 ff.).

${ }^{89}$ Thünken, GRUR-Prax 2017, 483.
} 
2. Für die Reichweite des forum delicti commissi ist auch die kollisionsrechtliche Rechtsprechung zur Bestimmung des Deliktsstatuts bei Verletzung von Rechten des geistigen Eigentums heranzuziehen. Zur Rom II-VO besteht Auslegungskonkordanz.

3. Art. 8 I Rom II-VO verweist auf das Recht des Staats, in dem der Schutz beansprucht wird (lex loci protectionis) und verwirklicht damit das allgemein anerkannte Schutzlandprinzip. Dem entspricht das sachrechtliche Territorialitätsprinzip. Daneben bedarf es für die Anwendbarkeit des nationalen Markenrechts eines „hinreichend wirtschaftlich relevanten Inlandsbezugs“, um die EU-Dienstleistungsfreiheit nicht zu beschränken.

4. Der zuständigkeitsrechtliche Deliktsort bei der Verletzung von Immaterialgüterrechten unterliegt dem Ubiquitätsprinzip. Danach versteht man unter dem „Ort des schädigenden Ereignisses“" sowohl den Ort, an dem der Schaden eingetreten ist als auch den Ort des ursächlichen Geschehens. Auch bei grenzüberschreitenden Markenrechtsverletzungen gilt der Grundsatz, dass der Handlungs- wie auch der Erfolgsort zuständigkeitsbegründend sind.

5. Bei geschäftsschädigenden Persönlichkeitsverletzungen durch cyber mobbing („Bolagsupplysningen“) besteht ein forum delicti commissi: (1) an der Niederlassung des Urhebers der Website-Inhalte (Handlungsort); (2) am Mittelpunkt der Interessen des Verletzten (Erfolgsort 1; ,qualifizierter Erfolgsort“); (3) an dem Ort, wo ein im Internet veröffentlichter Inhalt zugänglich ist oder war (Erfolgsort 2), mit Beschränkung der Kognitionsbefugnis auf den dort anteilig eingetretenen Schaden (Mosaikbetrachtung).

6. In der Entscheidung „Bolagsupplysningen“ ging es um die Rufschädigung einer juristischen Person über das Internet (cyber mobbing). Hier spricht einiges dafür, für die Lokalisierung des Erfolgsortes 1 („,qualifizierter Erfolgsort“) im Wege der rechtsaktübergreifenden Auslegung auf die umfangreiche Rechtsprechung und Literatur zur Lokalisierung des insolvenzrechtlichen Centre of Main Interests (COMI) zurückzugreifen.

7. Im „Abrufstaat“ als Teilerfolgsort (Erfolgsort 2) besteht bei Persönlichkeitsverletzungen nur eine beschränkte Kognitionsbefugnis für den dort eingetretenen Teilschaden.

8. Diese für Persönlichkeitsverletzungen entwickelten Grundsätze sind für die Bestimmung des Deliktsorts bei der Markenverletzung nur bedingt geeignet (Urteil „Wintersteiger"). Erfolgsort und Handlungsort sind hier anders zu lokalisieren. Zuständig für die Entscheidung über Schadensersatz- und Unterlassungsansprüchen des Markenrechtsinhabers sind die Gerichte des Mitgliedstaats, in dem die Marke eingetragen ist (deliktischer Erfolgsort) wie auch die Gerichte desjenigen Mitgliedstaats, in dem der angebliche Verletzer niedergelassen ist (deliktischer Handlungsort). Dagegen besteht wegen des Territorilitätsprinzips - oben Nr. 3 hier kein qualifizierter Erfolgsort (Erfolgsort 1; einheitlicher Erfolgsort) am Interessenmittelpunkt des Schutzrechtsinhabers.

9. Dem Urteil „Hanssen/ Knipping“ lassen sich Kriterien für die Abgrenzung des forum delicti commissi von der ausschließlichen Zuständigkeit des Registerstaats bei Streitigkeiten über gewerbliche Schutzrechte (Art. 24 Nr. 4 Brüssel 
Ia-VO) entnehmen. Danach unterliegt ein Prätendentenstreit grundsätzlich nicht der Zuständigkeit des Registerstaats. Diese Regel wird dem Grundsatz der engen Auslegung der ausschließlichen Zuständigkeiten (oben Nr. 1) gerecht, erscheint aber mit der vom $E u G H$ vertretenen Erstreckung des Art. 24 Nr. 4 Brüssel Ia-VO auf Nichtigkeitseinreden im Verletzungsprozess kaum vereinbar.

Open Access Dieses Kapitel wird unter der Creative Commons Namensnennung 4.0 International Lizenz (http://creativecommons.org/licenses/by/4.0/deed.de) veröffentlicht, welche die Nutzung, Vervielfältigung, Bearbeitung, Verbreitung und Wiedergabe in jeglichem Medium und Format erlaubt, sofern Sie den/die ursprünglichen Autor(en) und die Quelle ordnungsgemäß nennen, einen Link zur Creative Commons Lizenz beifügen und angeben, ob Änderungen vorgenommen wurden.

Die in diesem Kapitel enthaltenen Bilder und sonstiges Drittmaterial unterliegen ebenfalls der genannten Creative Commons Lizenz, sofern sich aus der Abbildungslegende nichts anderes ergibt. Sofern das betreffende Material nicht unter der genannten Creative Commons Lizenz steht und die betreffende Handlung nicht nach gesetzlichen Vorschriften erlaubt ist, ist für die oben aufgeführten Weiterverwendungen des Materials die Einwilligung des jeweiligen Rechteinhabers einzuholen.

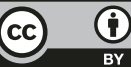

